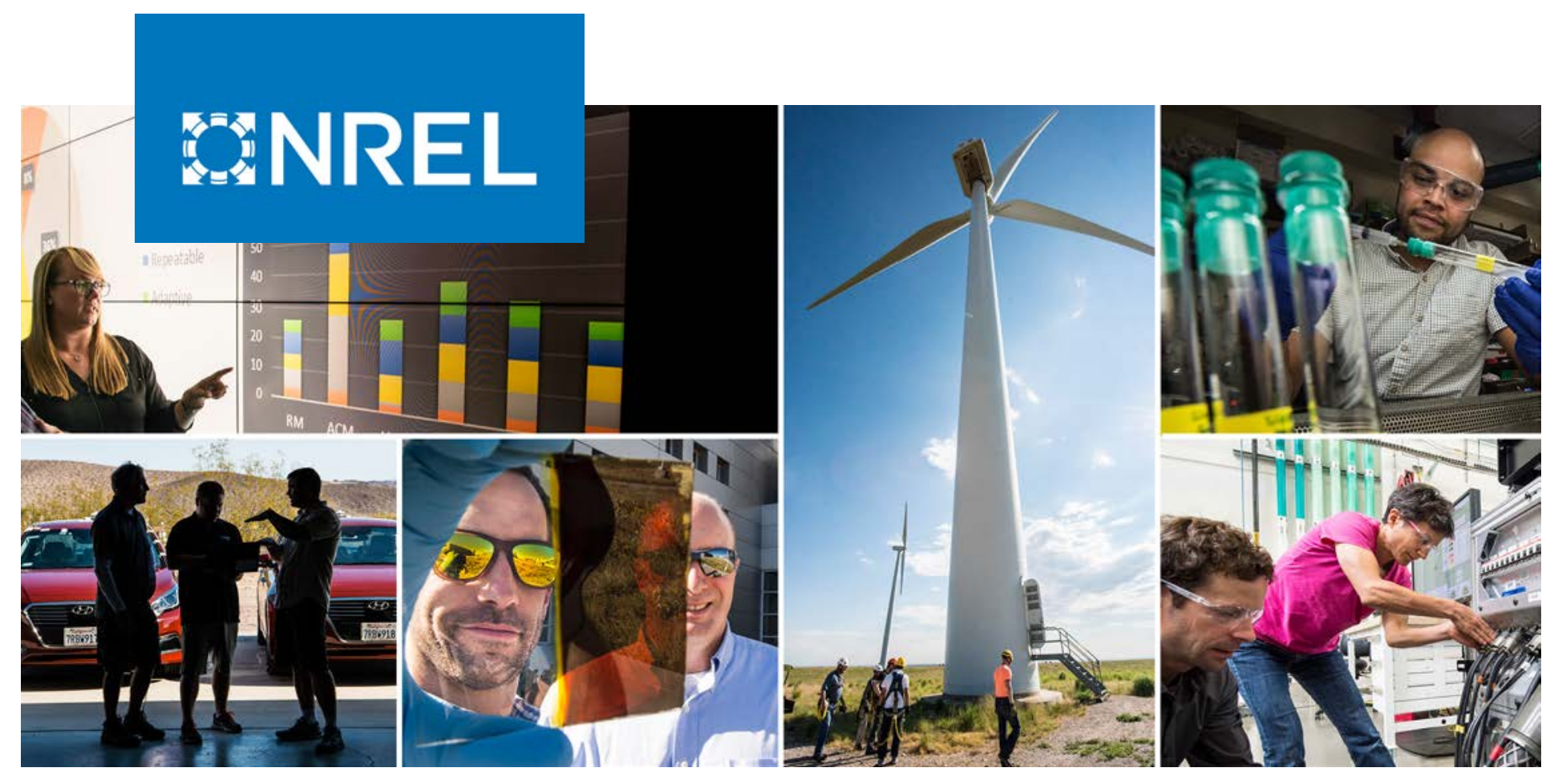

\title{
Wind Turbine Rotor Design Optimization Using Importance Sampling
}

\section{Preprint}

Ignas Satkauskas, Pietro Bortolotti, Evan Gaertner, Garrett E. Barter, Ryan King, and Peter A. Graf

National Renewable Energy Laboratory

Presented at the AIAA SciTech Forum and Exposition

Orlando, Florida

January 6-10, 2020

NREL is a national laboratory of the U.S. Department of Energy

Office of Energy Efficiency \& Renewable Energy

Operated by the Alliance for Sustainable Energy, LLC

This report is available at no cost from the National Renewable Energy Laboratory (NREL) at www.nrel.gov/publications. 


\title{
GNREL
}

\section{Wind Turbine Rotor Design Optimization Using Importance Sampling}

\section{Preprint}

\author{
Ignas Satkauskas, Pietro Bortolotti, Evan Gaertner, \\ Garrett E. Barter, Ryan King, and Peter A. Graf
}

National Renewable Energy Laboratory

\section{Suggested Citation}

Satkauskas, Ignas, Pietro Bortolotti, Evan Gaertner, Garrett E. Barter, Ryan King, and Peter A. Graf. 2020. Wind Turbine Rotor Design Optimization Using Importance Sampling: Preprint. Golden, CO: National Renewable Energy Laboratory. NREL/CP2C00-75555. https://www.nrel.gov/docs/fy20osti/75555.pdf.

NREL is a national laboratory of the U.S. Department of Energy Office of Energy Efficiency \& Renewable Energy Operated by the Alliance for Sustainable Energy, LLC

This report is available at no cost from the National Renewable Energy Laboratory (NREL) at www.nrel.gov/publications.

Contract No. DE-AC36-08GO28308
Conference Paper NREL/CP-2C00-75555 March 2020

National Renewable Energy Laboratory 15013 Denver West Parkway Golden, CO 80401 303-275-3000 • www.nrel.gov 


\section{NOTICE}

This work was authored by the National Renewable Energy Laboratory, operated by Alliance for Sustainable Energy, LLC, for the U.S. Department of Energy (DOE) under Contract No. DE-AC36-08GO28308. Funding provided by U.S. Department of Energy Office of Energy Efficiency and Renewable Energy Wind Energy Technologies Office. The views expressed herein do not necessarily represent the views of the DOE or the U.S. Government.

This report is available at no cost from the National Renewable Energy Laboratory (NREL) at www.nrel.gov/publications.

U.S. Department of Energy (DOE) reports produced after 1991 and a growing number of pre-1991 documents are available free via www.OSTI.gov.

Cover Photos by Dennis Schroeder: (clockwise, left to right) NREL 51934, NREL 45897, NREL 42160, NREL 45891, NREL 48097, NREL 46526.

NREL prints on paper that contains recycled content. 


\title{
Wind Turbine Rotor Design Optimization Using Importance Sampling
}

\author{
Ignas Satkauskas*, Pietro Bortolotti ${ }^{\dagger}$, Evan Gaertner ${ }^{\ddagger}$, Garrett E. Barter ${ }^{\S}$, Ryan King ${ }^{\mathrm{II}}$, and Peter A. Graf ${ }^{\|}$ \\ National Renewable Energy Laboratory, Golden, CO, 80401
}

\begin{abstract}
Probabilistic methods are commonly adopted to estimate both ultimate and fatigue loads in wind turbines, and multiple papers have presented a variety of approaches. In contrast, system-level, integrated design optimization methods for wind turbines have so far relied on deterministic methods to estimate loads and deflections. This work aims at addressing this gap by adopting importance sampling to estimate ultimate blade deflection for use within a rotor design optimization. A comparison between the results of this approach against traditional approaches using deterministic load evaluations show that the probabilistic approach is more conservative and that the solutions found with deterministic approaches violate the probabilistic constraint on tip deflection between $13 \%$ and 17\%. This highlights the importance of accurate load and deflection estimates and demonstrates that probabilistic approaches can be successfully integrated within a wind turbine design process despite the higher computational costs.
\end{abstract}

\section{Introduction}

In the past years, ultimate and fatigue load estimation for wind turbines has been approached with probabilistic-based methods [1-4]. To estimate ultimate loads, these methods combine multiple simulations of an aeroelastic solver initiated with different wind time histories and an extrapolation scheme to estimate probabilities of occurrence as low as $3.8 \times 10^{-7}$, which is equivalent to an event that occurs on average once in 50 years (assuming 10-minute reference period). Although superior in load estimation accuracy to the deterministic approaches, the family of probabilistic approaches, which commonly employ Monte Carlo sampling, is however characterized by two major drawbacks. First, the methods incur large computational costs, with thousands of load simulations required, necessitating the use of powerful workstations or high-performance clusters. Secondly, the outputs have a high sensitivity to the parameters of the extrapolation scheme, and the load predictions may be affected by a large uncertainty. Recent research efforts have addressed this challenge by investigating alternative approaches, such as importance sampling techniques [1-3].

Load analysis is a crucial step in every wind turbine design process and accurate load estimates are the basis for any successful design optimization. While in the past load analysis has mostly been a verification step, the current trend of developing holistic approaches for the design of the machines has integrated this step into iterative optimization loops. Following the need to limit the complexity and computational costs of the load assessment, deterministic load estimation approaches have however been typically adopted. Designers can still run probabilistic load assessment once an optimized design is identified via the deterministic approaches, but mismatches in the design margins can only be fixed manually when constraints are violated or ignored when conservative assumptions are taken.

This work aims at addressing this gap by integrating techniques for load estimation by adopting importance sampling within a holistic wind turbine design framework. The Wind-Plant Integrated System Design \& Engineering Model (WISDEM), which is a multidisciplinary design, analysis, and optimization framework developed at the National Renewable Energy Laboratory (NREL) [5], is adopted in this study.

This study considers three different approaches to ultimate load estimation and uses each in a design optimization loop to understand the impacts on blade design. The three approaches are:

1) Low Fidelity: Steady-state blade element momentum (BEM) solver with an extreme wind condition.

2) Traditional: Time-domain simulation of turbulent inflow conditions at multiple wind speeds and with Monte Carlo (MC) sampling of turbulent seeds, combined with the moment-based fitting extrapolation scheme.

\footnotetext{
*Postdoctoral Researcher, Computational Science Center

$\dagger$ Researcher, National Wind Technology Center

${ }^{\ddagger}$ Postdoctoral Researcher, National Wind Technology Center

${ }^{\S}$ Senior Engineer, National Wind Technology Center

"IS Senior Scientist, Computational Science Center

" Senior Scientist, Computational Science Center
} 
3) Importance Sampling (IS): Biased sampling of the turbulent temporal coherence to generate extreme load gusts directly coupled with extrapolation techniques.

Next, Section $[\mathrm{I}$ elaborates on the three approaches and Section $[\mathrm{II}$ compares the three sets of results. The main conclusions and the outlook on future work are reported in Section IV.

\section{Design Approaches}

The design of wind turbine rotors can be performed at various fidelity levels depending on the goal of the study. In this project, we define a simple aerostructural design optimization problem for the rotor of the NREL 5MW reference wind turbine [6]. The mathematical formulation of the optimization problem has the form:

$$
\begin{array}{rl}
\min _{\boldsymbol{c}, \boldsymbol{\theta}, \boldsymbol{s}, T S R} & L C o E(\boldsymbol{c}, \boldsymbol{\theta}, \boldsymbol{s} \boldsymbol{c}, T S R) \\
\text { s. t. } & X^{1 y r}(\boldsymbol{c}, \boldsymbol{\theta}, \boldsymbol{s} \boldsymbol{c}, T S R)=0.7 T C \\
& c_{i}^{\text {min }} \leq c_{i} \leq c_{i}^{\text {max }}, \quad i \in\{3,4,5\} \\
& \theta_{i}^{\text {min }} \leq \theta i \leq \theta_{i}^{\max }, \quad i \in\{3,4,5,6\} \\
& s c_{i}^{\text {min }} \leq s c_{i} \leq s c_{i}^{\text {max }}, \quad i \in\{3,4,5\} \\
& T S R^{\text {min }} \leq T S R \leq T S R^{\text {max }},
\end{array}
$$

where $L C o E$ represents the levelized cost of energy to be minimized, TSR the blade tip speed ratio, which is defined as $T S R=\frac{\Omega R}{V}$, where $\Omega$ is the rotor speed, $R$ the rotor radius, and $V$ the undisturbed wind speed, and $\boldsymbol{c}, \boldsymbol{\theta}$, and $\boldsymbol{s} \boldsymbol{c}$ denote vectors of six chord, twist, and spar caps thickness values defined from root to tip along blade span at the nondimensional positions $0.0,0.02,0.23,0.49,0.74$, and 1.0. A shape preserving spline interpolation scheme is adopted to reconstruct the spanwise distributions given the six control points. The optimization problem is formulated to not have all six entries of $\boldsymbol{c}, \boldsymbol{\theta}$, and $\boldsymbol{s} \boldsymbol{c}$ as active design variables, but only third, fourth, and fifth for $\boldsymbol{c}$ and $\boldsymbol{s} \boldsymbol{c}$, and third, fourth, fifth, and sixth for $\boldsymbol{\theta}$, to limit the optimizer to a solution space characterized by designs that are realistic and can be manufactured. For example, the blade should have spar caps that taper down to zero thickness towards blade root $\left(s c_{1}\right.$ and $\left.s c_{2}\right)$ and blade tip $\left(s c_{6}\right)$, blade root diameter $\left(c_{1}\right.$ and $\left.c_{2}\right)$ is defined via detailed three dimensional finite element studies and cannot be optimized, chord at blade tip $\left(c_{6}\right)$ is set to low values to simplify the construction process, and finally the aerodynamic twist of the cylindrical sections of blade root $\left(\theta_{1}\right.$ and $\left.\theta_{2}\right)$ is meaningless. The active design variables are also subjected to minimum and maximum bounds (3)-(6) to ensure feasible values for $c_{i}, s c_{i}$, and $T S R$, which must be positive. Finally, the design optimization problem is subjected to an equality constraint (2) that imposes that the 1-year maximum blade tip deflection $X^{1 y r}$ must be equal to $70 \%$ of the tower clearance (TC), namely 7.7 meters. Notably, this can also be defined as an inequality constraint (lower than $70 \%$ of $T C$ ), however the equality constraint is here preferred as it facilitates convergence in presence of the fairly flat solution space of $L C o E$ [7].

The merit function $L C o E$ is defined as

$$
L C o E=\frac{I C C \cdot A+O p e x}{A E P}
$$

where $A$ is the amortization factor, $O p e x$ is the annual operational expenditures, ICC is the initial capital cost, and $A E P$ is the annual energy production. For this optimization problem, $A$ is set equal to 0.07 and Opex is set equal to $\$ 144 / \mathrm{kW}$. The $I C C$ is computed from the equation

$$
I C C=B M \cdot k+T C C w b+B o S
$$

where $B M$ is blade mass, $k$ is a semi-empirical cost coefficient set equal to $\$ 14.6 / \mathrm{kg}, T C C w b$ is the cost of the machine without the blades and $B o S$ is the balance of station costs (inclusive of financial costs). For the sake of simplicity, TCC $w b$ and $B o S$ are held constant and equal to $\$ 1429 / \mathrm{kW}$ and $\$ 2979 / \mathrm{kW}$, which are the values reported for a fixed-bottom offshore reference project in the latest LCoE cost review from Stehly et al. [8].

The design problem (17)-(6) is solved using the models implemented in WISDEM, which is an open-source framework (github.com/WISDEM) for wind energy systems engineering, built upon the OpenMDAO library [9]. WISDEM has been historically comprised of fairly low fidelity models, and blade loads are by default estimated using the steady-state blade element momentum (BEM) theory solver, CCBlade. The loads are passed to the 1-D beam solver, pBeam, to 
Table 1 Models for the various disciplines adopted in the three design approaches.

\begin{tabular}{l|ccc}
\hline Discipline & Design 1 & Design 2 & Design 3 \\
\hline \hline Wind field & Steady with shear & Turbulent (pyTurbSim) \\
Aerodynamics & Steady-state BEM (CCBlade) & Time-resolved BEM (OpenFAST AeroDyn) \\
Structural dynamics & Beam model (pBeam) & Beam model (OpenFAST Elastodyn) \\
Controls & None & PI controller (OpenFAST Servodyn) \\
Statistical sampline & None & MC with extrapolation IS with extrapolation \\
\hline
\end{tabular}

compute the associated blade deflections and pBeam models the elastic properties of the blades using the 2-D cross sectional solver PreComp, which also estimates strains along the span of the blade. The blade loading is also used to estimate the rotor efficiency and derive a regulation trajectory that results in an estimated $A E P$. The WISDEM module linking these analysis tools together is called RotorSE. Strains and deflections can be posed as constraints in a design optimization with design variables parametrizing blade outer shape and inner structure. The outputs of RotorSE, $B M$ and $A E P$, are passed to the cost module, PlantFinanceSE, which estimates the values of ICC and $L C o E$.

WISDEM has an additional module, AeroElasticSE, that wraps around the open-source aeroservoelastic code, OpenFAST (formerly known as FAST) [10]. AeroElasticSE automatically generates multiple turbulent wind grids and OpenFAST input files, executes OpenFAST, and post-processes the outputs. Load and deflection time histories can be extracted from AeroElasticSE, passed into an extrapolation scheme, and used to compute a variety of quantities of interest. Due to the higher fidelity of OpenFAST, these outputs are of greater accuracy than those generated by CCBlade and pBeam. The outputs of AeroElasticSE are tied to the other WISDEM modules, including RotorSE, to enable use of OpenFAST in a design optimization loop.

When OpenFAST is used within the design loop it not only gives a better aeroelastic estimation of blade deflections, but also incorporates the behavior of the wind turbine controller. In this study, the standard PI controller of the NREL $5 \mathrm{MW}$ wind turbine [6] is used to control generator torque and blade pitch, and the controller logic is expanded to include a shut-down procedure, which estimates the wind speed from the filtered blade pitch angle. The controller imposes a turbine shut-down when the estimated wind speed goes beyond the cut-out wind speed of $25 \mathrm{~m} / \mathrm{s}$. This behavior is crucial to estimate realistic values of ultimate loads and deflections and will be important to understanding the results presented below.

In this study, we solve the minimization problem (1)-(6) adopting three different approaches to calculate maximum tip deflection $X^{1 y r}$ in (2). These are described in the next subsections and the models used in each approach are listed in Table 1. For all three approaches, the chosen optimization solver is the gradient-based solver CONMIN, which is implemented within the OpenMDAO plugin, pyOptSparse [11].

\section{A. Design 1: steady-state BEM}

In Design 1, we adopt the default configuration of RotorSE and estimate $X^{1 y r}$ by running a steady-state simulation where a single blade is exposed to a wind speed that corresponds to the peak wind speed observed during the extreme operating gust defined in the design load case (DLC) 2.3 of the IEC standards [12]. For the NREL 5MW, the wind speed is equal to $20 \mathrm{~m} / \mathrm{s}$ and the blade is set to rotate at the rated $\Omega$ with zero pitch angle. This approach clearly misses the aeroelastic physics and the behavior of a controller, but has the advantage of being computationally efficient.

\section{B. Design 2: IEC extrapolation}

A more rigorous approach to estimate maximum tip deflection $X^{1 y r}$ is followed in Design 2. Here, we follow the extrapolation procedure described in the Annex F of the IEC 61400-1 standards [12]. The procedure, which can be used to estimate both ultimate loads and deflections, consists of running six 10-minute OpenFAST simulations at equally spaced wind speed bin centers $\left\{V_{j}\right\}=\{2 j \mid j=2,3, . ., 12\} \mathrm{m} / \mathrm{s}$. Each of the six simulations is initialized with turbulent wind grids generated by pyTurbSim using six random seeds. pyTurbSim is a Python version [13] of the well-known wind model TurbSim [14]. Extreme values of tip deflection, $\left\{X_{i}\right\}$, are extracted from each simulation using the "peak over the threshold" method, which assumes that for a given time series with mean, $\mu$, and standard deviation, $\sigma$, peaks are selected as maximum values between two successive upcrossings of the threshold value $\mu+1.4 \sigma$. Figure 1 shows peak values found during a snapshot of one of the OpenFAST simulations in $V_{j}=12 \mathrm{~m} / \mathrm{s}$ wind speed bin. 


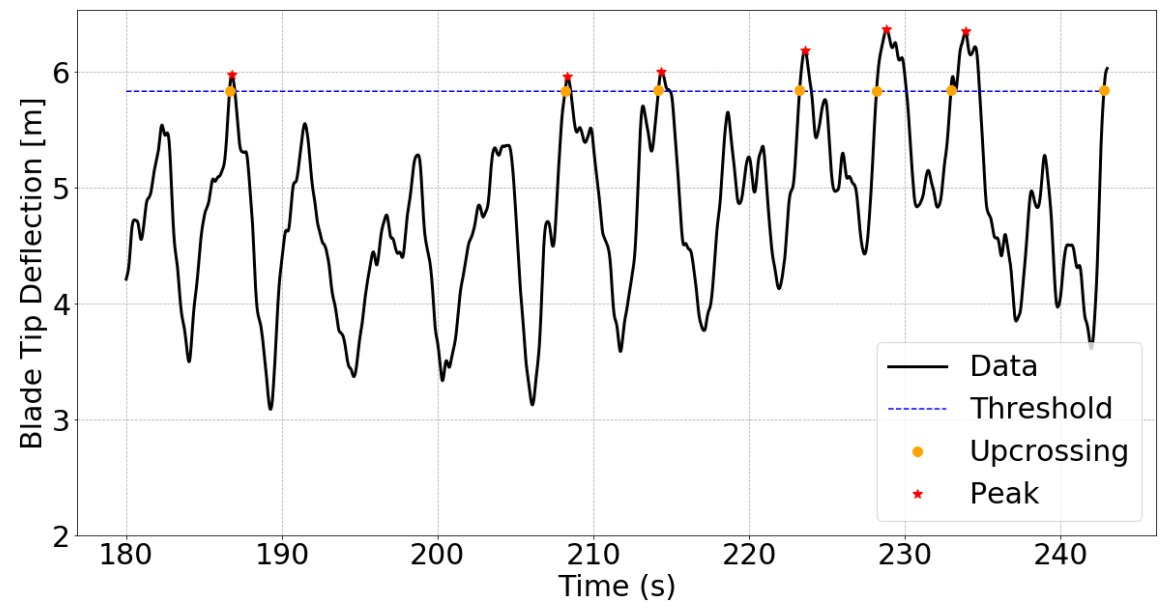

Fig. 1 Out-of-plane tip deflection peaks found during the fourth minute of a 10-minute OpenFAST simulation in $V_{j}=12(\mathbf{m} / \mathbf{s})$ wind speed bin.

Peak values of the tip deflection obtained from the OpenFAST simulations are used to find a local fit to a Weibull distribution for each wind speed, $V_{j}$. We choose a three-parameter Weibull distribution that has the cumulative density function (CDF) defined as:

$$
F_{j}\left(x ; c, x_{0}, \lambda\right)=1-\exp \left[-\left(\frac{x-x_{0}}{\lambda}\right)^{c}\right],
$$

where parameters $x_{0}>0, \lambda>0$, and $c>0$ are the location, scale, and shape parameters respectively. To obtain an analytic CDF from the dataset, we use the moment-based fitting procedure suggested by IEC 61400-1 and described in Moriarty et al [4]. First, we compute three moments of each data set (peaks extracted from six 10-minute OpenFAST simulations for wind speed $V_{j}$ ): mean $\mu$, standard deviation $\sigma$, and skewness $\eta$. The parameters describing the local Weibull distribution $F_{j}$ are obtained from the following equations:

$$
\begin{aligned}
& \eta=\frac{\frac{3}{c} \Gamma\left(\frac{3}{c}\right)-3\left(\frac{2}{c} \Gamma\left(\frac{2}{c}\right)\right)\left(\frac{1}{c} \Gamma\left(\frac{1}{c}\right)\right)+2\left(\frac{1}{c} \Gamma\left(\frac{1}{c}\right)\right)^{3}}{\left(\frac{2}{c} \Gamma\left(\frac{2}{c}\right)-\left(\frac{1}{c} \Gamma\left(\frac{1}{c}\right)\right)^{2}\right)^{3 / 2}} \\
& \lambda=\frac{\sigma}{\left(\frac{2}{c} \Gamma\left(\frac{2}{c}\right)-\left(\frac{1}{c} \Gamma\left(\frac{1}{c}\right)\right)^{2}\right)^{1 / 2}} \\
& x_{0}=\mu-\frac{\lambda}{c} \Gamma\left(\frac{1}{c}\right),
\end{aligned}
$$

where $\Gamma$ is the gamma function. Note that the first equation requires a root finding method to solve for parameter $c$. Figure 2 shows local Weibull distributions $F_{j}$ for wind speeds $V_{j}=8$ and $V_{j}=12$ obtained using the moment based fitting technique. by

Assuming the Reyleigh wind speed distribution, the long term exceedance probability of deflection $X$ is then given

$$
P_{e}(X)=\sum_{j}\left(1-\left(F_{j}(X)\right)^{n_{j}}\right)\left(R\left(V_{j}-\frac{\Delta V_{j}}{2}\right)-R\left(V_{j}+\frac{\Delta V_{j}}{2}\right)\right),
$$

where $V_{j}$ is the wind speed bin center, $\Delta V_{j}$ the bin width, $n_{j}$ the number of peaks in a 10-minute OpenFAST simulation, and $R$ is the CDF of the Reyleigh distribution in (14), with $V_{\text {ave }}$ dependant on the IEC wind turbine class. The NREL $5 \mathrm{MW}$ reference turbine is designed for Class IB and $V_{\text {ave }}$ is therefore equal to $10 \mathrm{~m} / \mathrm{s}$.

$$
R(V)=1-\exp \left(-\pi\left(\frac{V}{2 V_{\text {ave }}}\right)^{2}\right)
$$




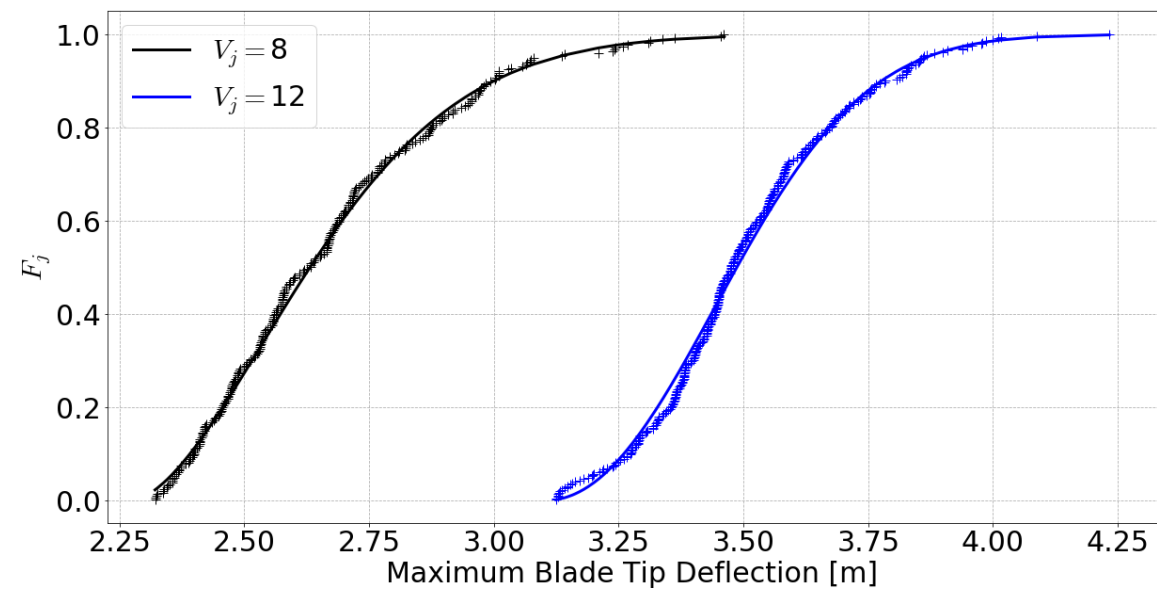

Fig. 2 Data points and analytic Weibull distributions $F_{j}$ for wind speeds $V_{j}=8$ and $V_{j}=12$ obtained using moment based fitting.

We obtain the value of 1-year maximum tip deflection from (13) by using a numerical root finding technique (bisection) and solving for $X^{1 y r}$ in:

$$
P_{e}\left(X^{1 y r}\right)=1.9 \times 10^{-5}
$$

for the 1-year recurrence period. Assuming the same 10-minute reference period and changing the right hand side of (15) to $1.9 \times 10^{-6}$ or $3.8 \times 10^{-7}$, one can obtain 10-year and 50-year recurrence period deflection values, $X^{10 y r}$ and $X^{50 y r}$, respectively.

\section{Design 3 : Importance Sampling}

In order to explain importance sampling used in Design 3, we first introduce the reader to the concept of temporal coherence. In both Design 2 and Design 3, OpenFAST simulations are initialized using turbulent wind grids generated by pyTurbSim. In Design 2, turbulent wind conditions are generated using the standard Veers model, as recommended in the IEC 61400-1, by computing inverse Fourier transform of spectral components with deterministic magnitudes and random phases. In the standard model, stochastic phases are independent and uniformly distributed between $-\pi$ and $\pi$. In Design 3, we employ a novel method of generating turbulent wind record introduced by Rinker et al [15], where random phases are computed as a cumulative sum of a sample of stochastic phase differences:

$$
\phi_{k+1}=\phi_{k}+\Delta \phi_{k}
$$

Here, $\phi_{k}$ is the phase of frequency $f_{k}$, and the phase difference $\Delta \phi_{k}$ is drawn from a circular wrapped Cauchy distribution given by:

$$
f\left(\Delta \phi_{k} ; \rho, \mu\right)=\frac{1}{2 \pi} \frac{1-\rho^{2}}{1+\rho^{2}-2 \rho \cos \left(\Delta \phi_{k}-\mu\right)}
$$

where $\mu \in[-\pi, \pi]$ and $\rho \in[0,1]$ are called location and concentration temporal coherence parameters, respectively. While the location parameter $\mu$ changes the location of the energy clustering, the concentration parameter $\rho$ controls the degree of non-stationarity in the time-domain realizations. Figure 3 shows the effect of the concentration parameter $\rho$ on turbulent wind time-domain realizations (in all three cases, $\mu$ is the same, $\mu=\pi$, so that the energy of the signal is concentrated in the middle of the 10-minute simulation). We note that using temporal coherence parameters $\mu=0$ and $\rho=0$ in (17) results in a uniform distribution, hence representing the standard turbulence model.

In addition to generating non-stationary turbulence, the notion of temporal coherence can be used in the analysis of non-stationarity in empirical wind records. In [15], Rinker et al analyzed wind data gathered at the Flatirons Campus of NREL in Boulder, Colorado, to estimate empirical values of the concentration parameter $\rho$ using 9,122 10-minute records. Each 10-minute record exhibited a slightly different value of $\rho$, and hence, the empirical distribution of $\rho$ itself was obtained. As it is shown in Graf et al [2], the distribution of empirical values of $\rho$ can be well approximated by a 


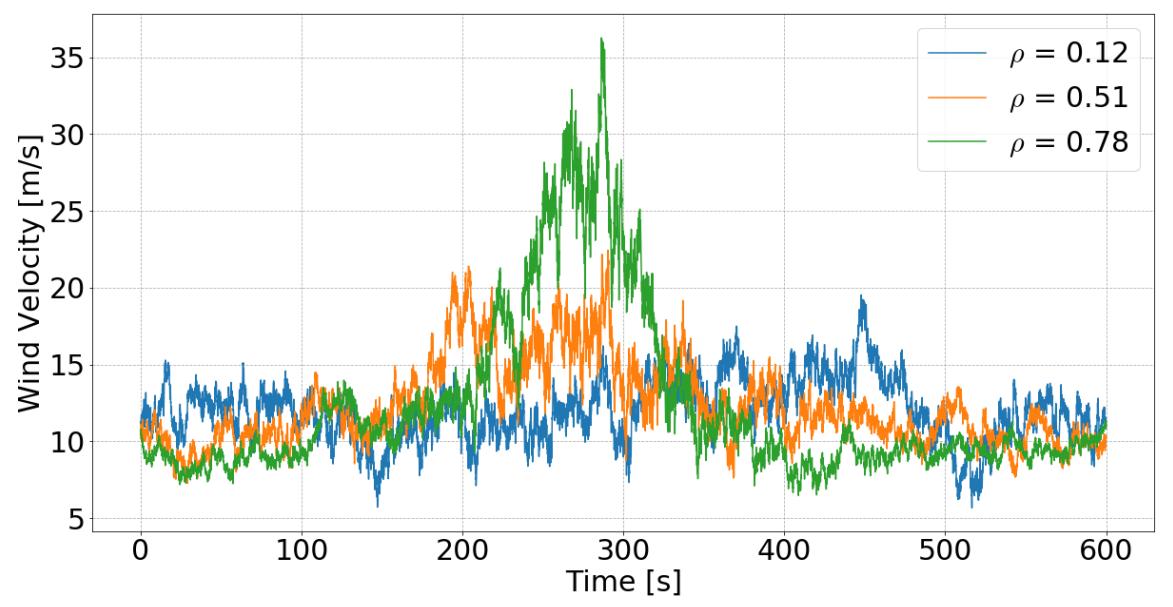

Fig.3 Wind velocity profile during 10-minute OpenFAST simulation using different values of the concentration parameter $\rho$ and a constant value of the location parameter $\mu=\pi$.

beta distribution $f(\rho ; a, b)$ defined in $(18)$ with parameters $a=2$ and $b=18$.

$$
f(\rho ; a, b)=\frac{1}{B(a, b)} \rho^{a-1}(1-\rho)^{b-1},
$$

In Design 3, to estimate tip deflection $X^{1 y r}$, we use $N_{s}=100$ samples of $\rho$ from the beta distribution (18) and a constant $\mu=\pi$ to generate 100 turbulent wind conditions with pyTurbSim. Each wind condition initializes 10-minute OpenFAST simulation from which we obtain one maximum of tip deflection $X_{i}$. We sort the set of obtained values $\left\{X_{i}\right\}_{i=1}^{N_{s}}$ from the smallest to the largest and use importance sampling to arrive at the desired 1-year maximum value $X^{1 y r}$.

In this way, temporal coherence is used to bias the otherwise random generation of turbulent wind fields to those that produce extreme gust events and ultimate loading cases. Importance sampling, in the general case, is explained by the following equation:

$$
E_{f}[y(\rho)]=\int y(\rho) f(\rho) d \rho=\int y(\rho) \frac{f(\rho)}{g(\rho)} g(\rho) d r=E_{g}\left[y(\rho) \frac{f(\rho)}{g(\rho)}\right],
$$

where $y(\rho)$ is an arbitrary function of random variable $\rho$, and $f$ and $g$ are called nominal and importance distributions, respectively. Recalling that probability can be expressed as expectation of an indicator function:

$$
P(A)=E[I(A)],
$$

with

$$
I(A)= \begin{cases}1, & \text { if A occurs } \\ 0, & \text { otherwise }\end{cases}
$$

and making use of sample average approximation

$$
E[h(\rho)] \approx \frac{1}{N_{s}} \sum_{i} h\left(\rho_{i}\right)
$$

we arrive at the discrete CDF 


$$
\begin{aligned}
P\left(X \leq X_{j}\right) & =E_{f}\left[I\left(X(\rho) \leq X_{j}\right)\right] \\
& =E_{g}\left[I\left(X(\rho) \leq X_{j}\right) \frac{f(\rho)}{g(\rho)}\right] \\
& =\frac{1}{N_{s}} \sum_{i} I\left(X\left(\rho_{i}\right) \leq X_{j}\right) \frac{f\left(\rho_{i}\right)}{g\left(\rho_{i}\right)} \\
& =\frac{1}{N_{s}} \sum_{i \leq j} \frac{f\left(\rho_{i}\right)}{g\left(\rho_{i}\right)},
\end{aligned}
$$

where, to obtain the last equality, we use the fact that the set of tip deflections $\left\{X_{i}\right\}$ is ordered from the smallest to the largest. Finally, we write the expression for discrete probability of exceedance:

$$
P_{e}\left(X_{j}\right)=1-P\left(X \leq X_{j}\right)
$$

Weights $w_{i}=f\left(\rho_{i}\right) / g\left(\rho_{i}\right)$ in $(26)$ are calculated using the nominal $f(\rho ; 2,18)$, and importance, $g(\rho ; 2,5)$ beta distribution (18) shown in Figure 4 Shifting the probability density towards higher values of $\rho$, i.e. smaller values of $b$ in (18), gives greater temporal coherence and the production of stronger gusts. Since, on average, weights $w_{i}$ are less than one, importance sampling can reach smaller probabilities of exceedance with fewer samples than standard Monte Carlo sampling, as seen in Figure 5. In Monte Carlo case, one can think of the weights $w_{i}$ in (26) as equal to one. Nevertheless, while probabilities of exceedance obtained using importance sampling are much smaller than the ones obtained using Monte Carlo $\left(10^{-4}\right.$ vs. $\left.10^{-2}\right)$, they are not small enough to reach the 1 -year return period probability of $1.9 \times 10^{-5}$. Hence, discrete values of CDF in (26) are used to fit an analytic Weibull distribution and the required probability levels are obtained by extrapolation. Since probabilities obtained using 100 importance samples reach $10^{-4}$, we are able to better represent the tail behaviour of the Weibull distribution and consequently be more confident in the extrapolation results. These extreme events also trigger the shutdown controller, causing a dynamic response of the machine that in Design 2 could only be captured by increasing the list of DLCs including the 2.X cases [12].

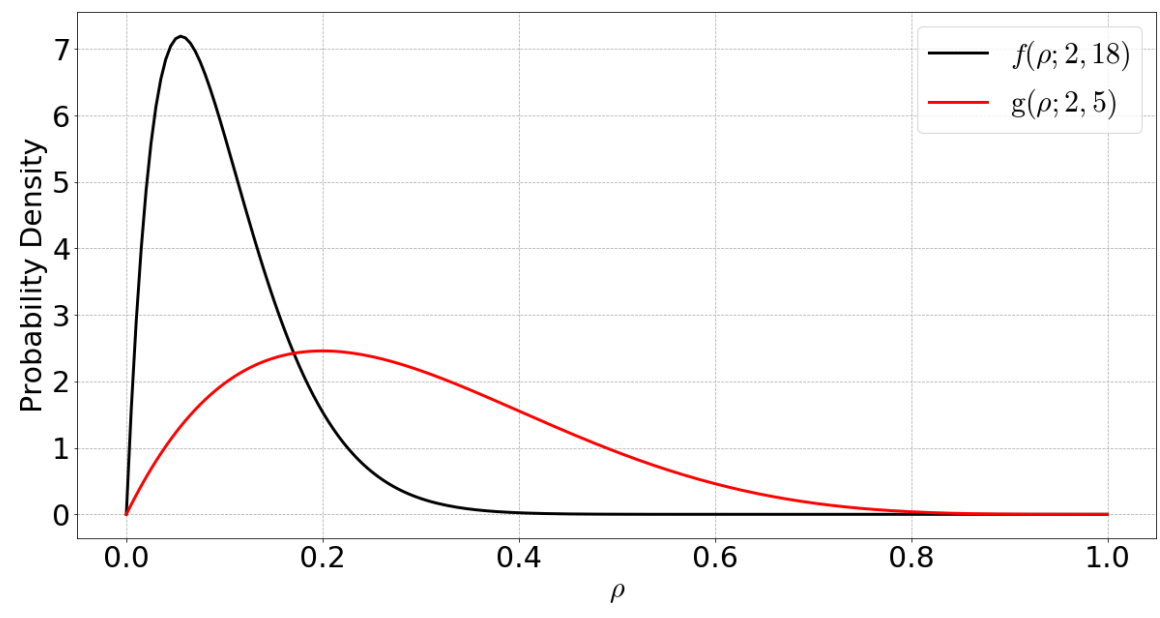

Fig. 4 Nominal, $f(\rho ; 2,18)$, and importance, $f(\rho ; 2,5)$, distributions of $\rho$.

\section{Results}

The results of the optimization runs from the design loop adopting steady-state BEM (Design 1), the design loop adopting OpenFAST and a standard extrapolation (Design 2), and the design loop adopting the combination of OpenFAST, importance sampling, and extrapolation (Design 3) are presented in this section. The optimizations were conducted on NREL's high performance cluster, Eagle. Parallelization is implemented using the open-source Python library MPI. For each iteration, 12 function evaluations are run (reference case plus 11 finite differences) for all three approaches, but the function evaluations have very different computational costs. Design 1 runs a single steady-state 


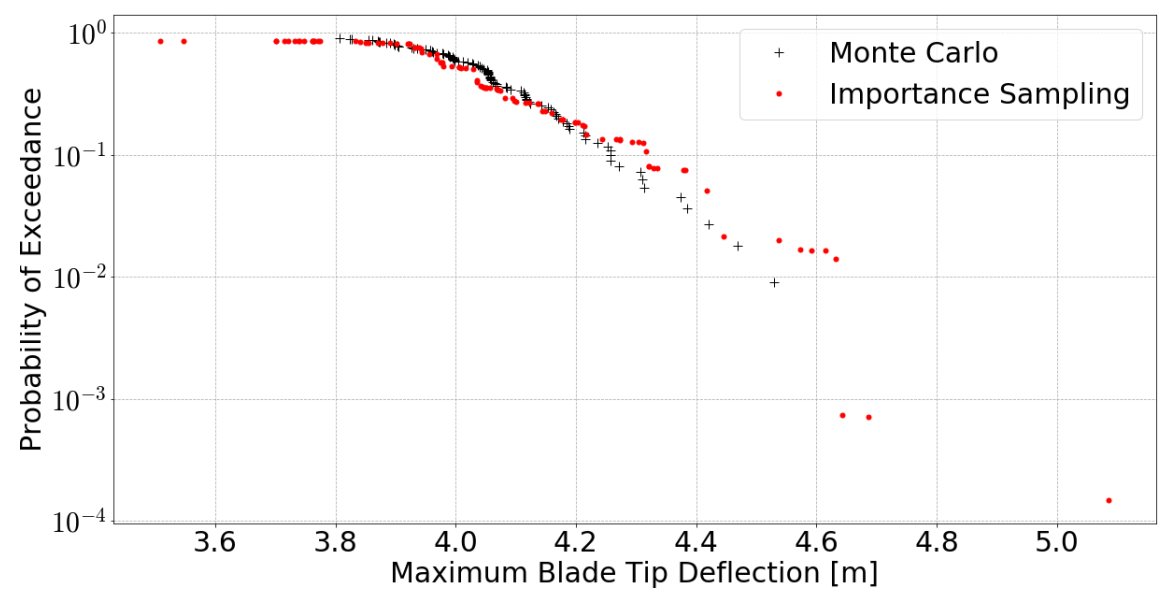

Fig. 5 Probability of exceedance calculated using standard Monte Carlo and Importance Sampling methods.

Table 2 Differences in the computational costs of designs 1-3.

\begin{tabular}{l|ccccc}
\hline & \# iterations & $\begin{array}{c}\text { \# of aeroelastic simulations } \\
\text { per iteration }\end{array}$ & $\begin{array}{c}\text { \# of HPC } \\
\text { nodes used }\end{array}$ & $\begin{array}{c}\text { \# of cores per } \\
\text { node called }\end{array}$ & $\begin{array}{c}\text { total time } \\
\text { in hours }\end{array}$ \\
\hline \hline Design 1 & 140 & 1 & 1 & 11 & 0.2 \\
Design 2 & 73 & 66 & 31 & 36 & 10 \\
Design 3 & 97 & 100 & 31 & 36 & 14 \\
\hline
\end{tabular}

simulation and the data post-processing in a few seconds, while Design 2 and 3 require 72 and 100 OpenFAST simulations respectively. Although Design 1 can be run serially, all three approaches are parallelized. Design 1 is parallelized across finite difference calls and runs on a single node in a matter of minutes. Design 2 and Design 3 are instead parallelized across both finite differences and OpenFAST aeroservoelastic simulations, and have a run time of approximately one day running on 31 nodes. Table 2 lists the main metrics summarizing the computational costs of the three design approaches.

Figure 6 shows the comparison of the solutions of the three design approaches against the initial design for chord, twist, and spar caps thickness distributions along blade span. Table 3 shows the relative differences of $A E P$, blade mass, TSR, and $L C o E$ of the design solutions in respect to the initial design. All three design approaches reduce the $L C o E$ of the initial design thanks to a change in the twist, which is found to be aerodynamically suboptimal, and a reduction in the spar caps thickness, which is overly conservative in the baseline design. The three design approaches land on roughly the same twist distribution, while differences exist in the chord and spar caps thickness distributions. The higher values of tip deflection predicted by importance sampling with extrapolation drive Design 3 toward thicker laminates and more moderate reductions in blade mass. The trade-off between aerodynamic and structural efficiency is also slightly different among the solutions, with Design 1 favoring a more tapered chord distribution and a higher rated $T S R$ resulting in higher $A E P$.

Overall, the design solution found by Design 3 using importance sampling and extrapolation is more conservative, and the reduction in LCoE is more limited than in the solutions generated by Design 1 and Design 2 using steady state and Monte Carlo methods, respectively. In fact, Design 1 and Design 2 violate the tip deflection constraint when estimated by importance sampling by $13 \%$ and $17 \%$ respectively. Given that importance sampling and Design 3 offer a higher degree of trust in the result, future work will focus on investigating the possibility of decreasing the safety factors, which might be reduced in the view of a site-specific design capability, where importance sampling is tuned to mimic the real wind conditions of a site.

\section{Conclusions}

This study integrates probabilistic techniques to estimate ultimate loads and deflections within a wind turbine rotor design procedure. A comparison is established among probabilistic and deterministic design approaches. The first 
Table 3 Differences in $T S R$, blade mass, $A E P$ and $L C O E$ between the initial design of the NREL 5MW and the optimized designs 1-3.

\begin{tabular}{c|cccc}
\hline \multirow{2}{*}{ Metric } & \multicolumn{4}{|c}{ Percentage change (\%) } \\
\cline { 2 - 5 } & $A E P$ & Blade mass & TSR & LCoE \\
\hline \hline Design 1 & 0.70 & -12.2 & 9.27 & -1.32 \\
Design 2 & 0.54 & -13.0 & 5.12 & -1.20 \\
Design 3 & 0.54 & -9.73 & 4.42 & -1.03 \\
\hline
\end{tabular}
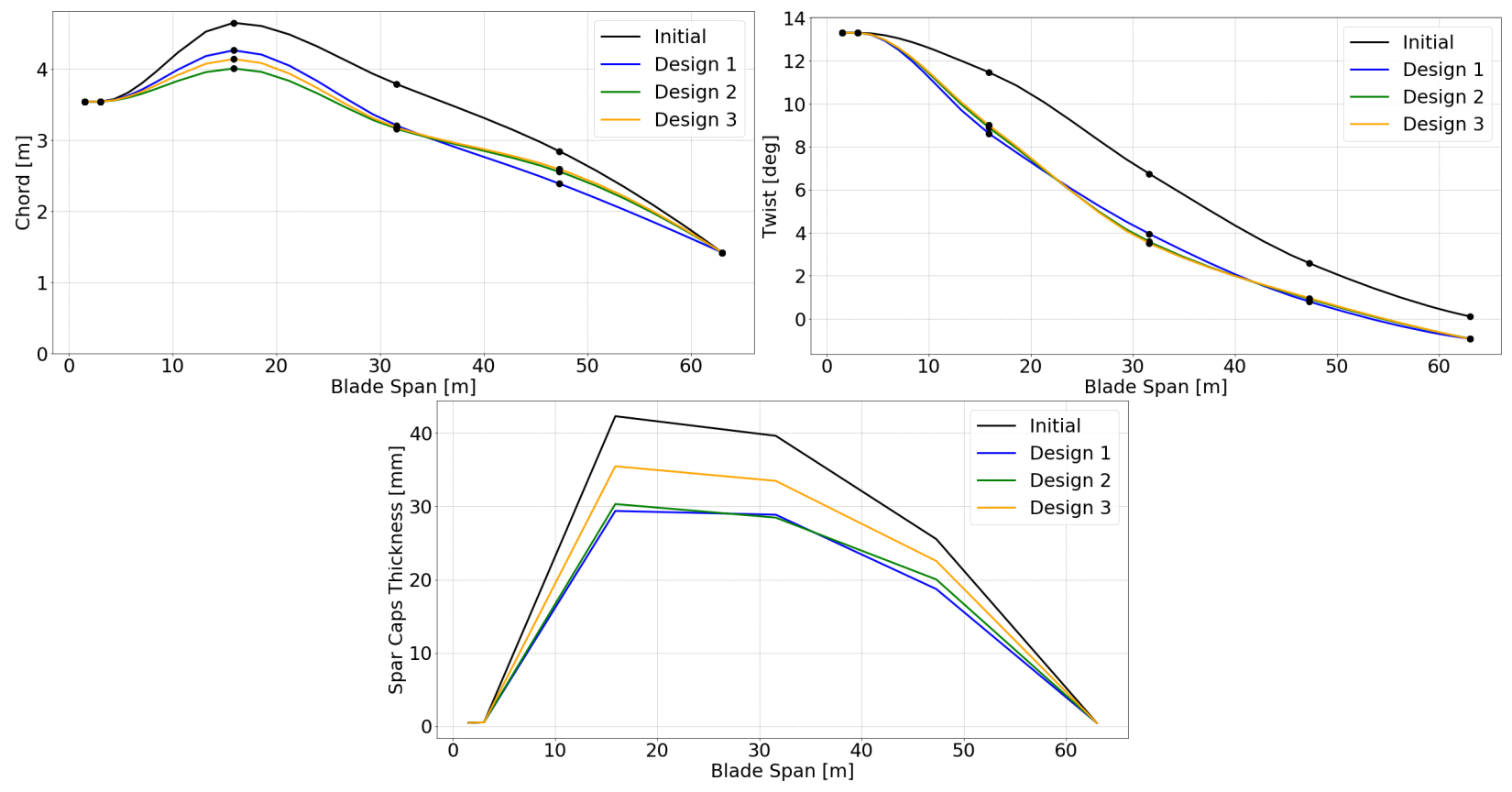

Fig. 6 Comparison of chord, twist, and spar caps along blade span for the initial design of the NREL 5MW and the solutions found in designs 1-3. 

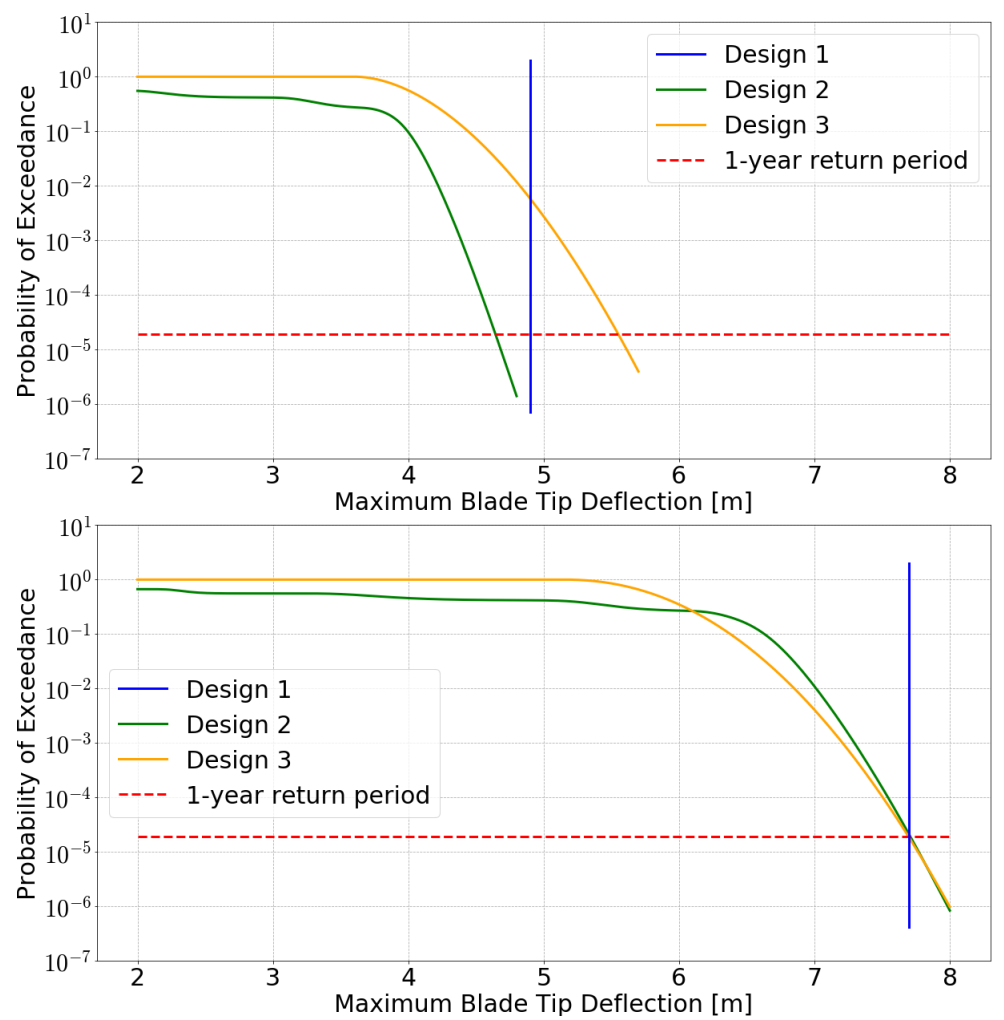

Fig. 7 Initial (top) and final (bottom) probability of exceedance of the ultimate blade tip deflection of the NREL 5MW wind turbine.

approach estimates the ultimate blade tip deflection via a steady-state aerodynamic solver, the second one adopts the combination of a full aeroservoelastic model and an extrapolation scheme, and the third one implements an importance sampling technique and an extrapolation scheme. The different values of blade tip deflections drive the blade optimizer towards different aerostructural design solutions and highlight the potential sub-optimality of design approaches adopting simplified load estimation procedures. This can be overcome by using partial safety factors, which are commonly found in current IEC guidelines. However, the prospect of moving beyond the need for safety factors and towards site-specific design is a tantalizing opportunity that could be opened up through the use of importance sampling and other probabilistic design methods in the future.

Future work will focus on expanding the design approaches to estimate a wider list of metrics via the combination of importance sampling and extrapolation. The goal will be to include probabilistic stress, strain, and fatigue loads into the design optimization using advanced sampling techniques.

\section{Acknowledgments}

This work was authored by the National Renewable Energy Laboratory, operated by Alliance for Sustainable Energy, LLC, for the U.S. Department of Energy (DOE) under Contract No. DE-AC36-08GO28308. Funding provided by the Wind Energy Technology Office. The views expressed in the article do not necessarily represent the views of the DOE or the U.S. Government. The U.S. Government retains and the publisher, by accepting the article for publication, acknowledges that the U.S. Government retains a nonexclusive, paid-up, irrevocable, worldwide license to publish or reproduce the published form of this work, or allow others to do so, for U.S. Government purposes.

The research was performed using computational resources sponsored by the Department of Energy's Office of Energy Efficiency and Renewable Energy and located at the National Renewable Energy Laboratory. 


\section{References}

[1] Graf, P. A., Dykes, K., Damiani, R., Jonkman, J., and Veers, P., "Adaptive stratified importance sampling: hybridization of extrapolation and importance sampling Monte Carlo methods for estimation of wind turbine extreme loads," Wind Energy Science, Vol. 3, No. 2, 2018, pp. 475-487. doi:10.5194/wes-3-475-2018, URL https://www . wind-energ-sci . net/3/475/2018/

[2] Graf, P. A., Satkauskas, I., King, R., Dykes, K., Quick, J., Kilcher, L., and Rinker, J., “Temporal Coherence Importance Sampling for Wind Turbine Extreme Loads Estimation," AIAA Scitech 2019 Forum, 2019. doi:10.2514/6.2019-1798, URL https://doi.org/10.2514/6.2019-1798

[3] Graf, P. A., Damiani, R., Dykes, K., and Jonkman, J. M., “Advances in the Assessment of Wind Turbine Operating Extreme Loads via More Efficient Calculation Approaches," AIAA Scitech 2017 Forum, 2017. doi:10.2514/6.2017-0680, URL https://doi.org/10.2514/6.2017-0680

[4] Moriarty, P., Holley, W., and Butterfield, S., "Effect of Turbulence Variation on Extreme Loads Prediction for Wind Turbines," Journal of Solar Energy Engineering, Vol. 124, 2002. doi:10.1115/1.1510137.

[5] Dykes, K., Ning, A., King, R., Graf, P., Scott, G., and Veers, P., "Sensitivity analysis of wind plant performance to key turbine design parameters: a systems engineering approach," Tech. rep., National Renewable Energy Laboratory, 2014.

[6] Jonkman, J., Butterfield, S., Musial, W., and Scott, G., "Definition of a 5-MW Reference Wind Turbine for Offshore System Development," Tech. rep., National Renewable Energy Laboratory, 2009.

[7] Bortolotti, P., Bottasso, C. L., and Croce, A., "Combined preliminary-detailed design of wind turbines," Wind Energy Science, Vol. 1, 2016. doi:https://doi.org/10.5194/wes-1-71-2016.

[8] Stehly, T., Beiter, P., Miller, D., and Scott, G., “2017 Cost of Wind Energy Review,” Tech. rep., National Renewable Energy Laboratory, 2018.

[9] Gray, J. S., Hwang, J. T., Martins, J. R. R. A., Moore, K. T., and Naylor, B. A., “OpenMDAO: An Open-Source Framework for Multidisciplinary Design, Analysis, and Optimization,” Structural and Multidisciplinary Optimization, 2019.

[10] “OpenFAST GitHub Repository,”, Jan 2019. URL https://github.com/OpenFAST/openfast

[11] Perez, R. E., Jansen, P. W., and Martins, J. R. R. A., “pyOpt: A Python-Based Object-Oriented Framework for Nonlinear Constrained Optimization," Structural and Multidisciplinary Optimization, Vol. 45, No. 1, 2012, pp. 101-118. doi:10.1007/ s00158-011-0666-3.

[12] IEC, “IEC: 61400-1, Wind turbines - Part 1: Design requirements," Tech. rep., 2005. URL https://webstore.iec.ch/ publication/22259.

[13] “pyTurbSim GitHub Repository,”, Nov 2019. URL https://github.com/lkilcher/pyTurbSim

[14] Veers, P., “Three-Dimensional Wind Simulation,” Tech. rep., Sandia National Laboratories, 1988.

[15] Rinker, J. M., Gavin, H. P., Clifton, A., Veers, P. S., and Kilcher, L. F., "Temporal Coherence: A Model for Nonstationarity in Natural and Simulated Wind Records," Boundary-Layer Meteorology, Vol. 159, No. 2, 2016 , pp. 373-389. doi:10.1007/s10546-015-0121-x, URL https://doi.org/10.1007/s10546-015-0121-x 